\title{
INTERNETWORKING TELEPHONY, IP AND ATM NETWORKS*
}

\author{
Malathi Veeraraghavan \\ Department of Electrical Engineering \\ Polytechnic University \\ 6 Metrotech Center \\ Brooklyn, NY 11210 \\ mv@poly.edu
}

Resumo - Este artigo propõe um princípio geral de interação de redes aplicável à ligação de quaisquer pares de redes. O princípio é aplicado ao problema específico de interligar redes ATM com a rede telefônica pública (PSTN) e redes com protocolo Internet (IP). Um protótipo demonstrativo é descrito para a interligação de redes IP e ATM.

Abstract - This paper proposes a general internetworking principle applicable to internetworking any two networks. We apply this principle to the specific problem of internetworking ATM networks with the PSTN and IP networks. A proof-of-concept prototype implementation of the IP-ATM internetworking solution is also described.

Keywords - Internetworking, ATM networks, PSTN, IP networks.

\section{INTRODUCTION}

The PSTN (Public Switched Telephone Network) and the Internet are wide-area networks that support a number of applications. These applications are of three types:

a. interactive applications, such as telephony, telnet and rlogin;

b. bulk-data applications, such as file transfers, electronic mail and web downloads;

c. streaming applications for audio and video from stored or live sources.

Ideally, one can associate the most suitable networking mode with each of these types of applications. We identify three types of networking modes:

a. circuit-switched (which implies connection-oriented),

b. connectionless (which implies packet-switched), and

c. packet-switched connection-oriented.

A network is connection-oriented if a connection is set up prior to data transfer, while it is connectionless if no such connection setup phase preceeds data exchange. A network is packet-switched if its nodes perform switching action based on bits in the packet header, while it is circuit-switched, if its nodes perform switching action based on the position of the

*This work was partially funded by the Advanced Technology Program of the National Institute of Standards and Technology, U.S. government. arriving bits, where position includes any combination of the space, time and wavelength dimensions.

Interactive applications generate bursty data and hence are best served by packet-switched networks. In addition, they have strict delay and delay jitter requirements, which implies the need for connection-oriented networking. Thus, packet-switched connection-oriented networks, such as ATM (Asynchronous Transfer Mode) networks are ideally suited for transporting data from interactive applications. Bulk-data applications could involve the exchange of small quantities of data or large quantities of data. If the file/email message/web page size is small in that the time to transfer the file is much smaller than the call setup delay, then the connectionless (which implies packet-switched) networking mode is the ideal mode. For large bulk-data transfers, where the time to transfer the file is much larger than the call setup delay, a circuit-switched network can be used since no packet header or acknowledgment overheads are incurred once the circuit is set up. On the other hand, connectionless networking with a reliable transport layer protocol, such as TCP/IP networking, can also be used, since in spite of the packet headers, TCPs congestion control mechanism has the ability to adapt the data rate to existing network conditions, which could result in a far shorter data transfer delay than in a circuit-switched network in which the data rate of the circuit remains unaltered after set up. Thus, both circuit-switched (connection-oriented) networks and connectionless (packetswitched) networks are suitable for large bulk-data transfers. Finally, streaming applications, in which audio or video are continuously streamed are best served with a circuitswitched network. Being connection-oriented, it provides delay and delay-jitter guarantees. Also, since the data is continuous, there are no silences and hence packet-switching is not required.

This understanding of the ideal networking modes for different types of applications leads us to believe that the goal of an integrated networking solution, as was intended with ATM, is not the right approach. Instead, different networks types should be made to coexist and interconnected by gateways that direct traffic from the networking mode in which data is generated to the ideal mode wherever possible. It is with this aim that we study the problem of how to internetwork ATM this networks with the PSTN and the Internet.

Telephony traffic is generated by PSTN endpoints as well as by Internet telephony PCs. However, we think a packetswitched connection-oriented mode such as ATM is the ideal 
mode for such traffic. Hence we propose gateways that can internetwork the PSTN and ATM networks, as well as the Internet and ATM networks to redirect such traffic.

We observe the analogy between networking techniques used to move audio, data and video traffic, and transportation networks used to move people. There are four major transportation networks, roadways, railways, airlines and shipping networks, to move people. Similarly, different networking technologies can exist to move information. A significant feature of transportation networks is that they coexist, in that, while predominantly, only roadways have access links to people, roads lead to airports that are essentially gateways to the airways transportation network. Using this analogy, we believe that having developed a new networking technology, i.e., ATM, if appropriate gateways are implemented, ATM technology will be most useful for information transfer even if ATM networks do not have direct access links.

This paper provides a solution to the more general internetworking problem, independent of the type of networks being internetworked. For example, the solution proposed in this paper could be used for internetworking the PSTN and the Internet. Internetworking is required to connect an endpoint in one network to an endpoint in another network, or when connecting two endpoints of the same network, if some segment of the communication path traverses a different network from the network to which the two endpoints are connected.

In Section 2, we state our assumptions for this work. We then present our general internetworking principle in Section 3. In Section 4, existing standards solutions for internetworking PSTNs and ATM networks, VTOA (Voice and Telephony Over ATM) [1], and IP networks and ATM networks, MPOA (MultiProtocol Over ATM) [2], are evaluated against

our general internetworking principle. The application of our proposed general internetworking solution to the specific problem of internetworking PSTNs and ATM networks, and IP networks and ATM networks, is described in Section 5. A prototype implementation of our proposed IP-ATM internetworking solution is described in Section 6.

\section{ASSUMPTIONS}

We make an assumption that all three networks shown in Fig. 1 either already support or will soon support both connectionoriented and connectionless networking modes. Connectionoriented networking requires a signaling protocol, a routing protocol and a user-plane protocol, while connectionless networking requires a datagram protocol (which is the userplane protocol) and a routing protocol. Both networking modes require an addressing scheme.

All three networks shown in Fig. 1 support both connection-oriented and connectionless networking modes. The protocols and addressing schemes used in these three networks are listed in Table 1. The acronyms used in Table 1 are spelled out at the end of the paper. SS7 (Signaling System No. 7) networks provide connectionless services to complement the connection-oriented service provided by

\begin{tabular}{|c|c|c|c|c|}
\hline & & \multicolumn{3}{|c|}{ Conection-Oriented Mode } \\
\hline & \multicolumn{2}{|c|}{ Conectionless Mode } & & \\
\hline $\begin{array}{l}\text { Node } \\
\text { (Adress) }\end{array}$ & $\begin{array}{l}\text { Datagram } \\
\text { Protocol }\end{array}$ & $\begin{array}{l}\text { Routing } \\
\text { Protocol }\end{array}$ & $\begin{array}{l}\text { Signaling } \\
\text { Protocol }\end{array}$ & $\begin{array}{l}\text { User- } \\
\text { Plane } \\
\text { Protocol }\end{array}$ \\
\hline $\begin{array}{l}\text { PSTN } \\
\text { Switch/STP } \\
\text { (E.164/ } \\
\text { Point } \\
\text { Code) }\end{array}$ & $\begin{array}{l}\text { SS7 } \\
\text { MTP L3 }\end{array}$ & $\begin{array}{l}\text { e.g., } \\
\text { RTNR } \\
\text { /SS7 pro- } \\
\text { visioning }\end{array}$ & $\begin{array}{l}\text { Q.931/ } \\
\text { ISUP }\end{array}$ & STM \\
\hline $\begin{array}{l}\text { IP Switch } \\
\text { Router } \\
\text { (IPv4/IPv6) }\end{array}$ & $\mathrm{IP}$ & $\begin{array}{l}\text { OSPF, } \\
\text { BGP, etc. }\end{array}$ & $\begin{array}{l}\text { RSVP, } \\
\text { IPv6 }\end{array}$ & $\mathrm{IP}$ \\
\hline $\begin{array}{l}\text { ATM } \\
\text { Switch } \\
\text { Router } \\
\text { (AESA) }\end{array}$ & CL-ATM & $\begin{array}{l}\text { PNNI } \\
\text { routing }\end{array}$ & \begin{tabular}{l|} 
UNI/ \\
PNNI \\
signaling
\end{tabular} & ATM \\
\hline
\end{tabular}

Table 1. Protocols and addressing schemes.

PSTNs. Many of the control-plane applications, such as the Intelligent Network (IN) based services and mobility management applications use the SS7 network for short query-response type transactions. The addressing scheme used in PSTNs, E.164 addressing, is different from the address used in the datagram protocol (MTP L3 protocol) in SS7 networks, i.e., point code addresses. This creates the need for address resolutions from E.164 addresses to point codes, also called Global Title Translations (GTT), whenever an application using one mode triggers a communication using the other mode. For example, 800-number translation requests, carried using the connectionless mode, are triggered by a telephone call, an application that uses the connectionoriented mode. The routing protocol used in PSTNs for SVC routing varies from one service provider to the next. For example, in the AT\&T network, RTNR (Real Time Network Routing), is used. Routing data for datagram forwarding is downloaded to STPs (Signaling Transfer Points) through management systems. In other words, a centralized operations support system computes routing data to reach various destinations (identified by point codes) in SS7 networks and downloads this data to the various STPs (datagram routers).

In IP networks, 4-byte IPv4 and 16-byte IPv6 addresses are used. Currently, the dominant mode of operation is the connectionless mode, which uses the IP datagram protocol and the OSPF/BGP (Open Shortest Path First/Border Gateway Protocol) family of routing protocols. The next generation IP networks, based on IPv6 [3], will support both networking modes. The IPv6 header has a flow label field that implies that IP routers will be equipped to maintain flow-based state, a characteristic of connection-oriented networking. In the signaling protocol column of Table 1 , we listed both RSVP (Resource reSerVation Protocol) [4] and IPv6 because IPv6 supports the hop-by-hop option for establishing a flow when the first IPv6 packet in a flow appears at a router. We labeled the network node of the Internet an IP switch router in Fig. 1 to signify that it supports both networking modes. A significant point to note is that 


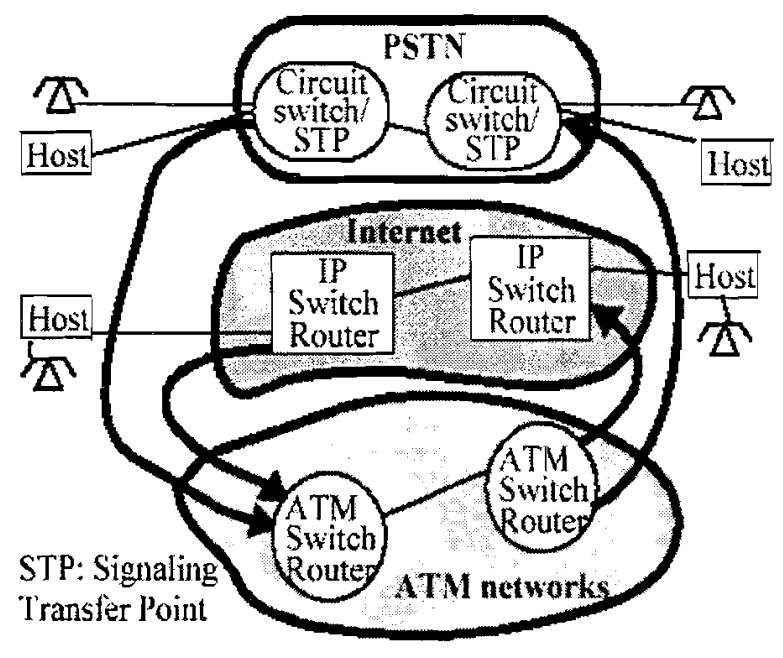

Figure 1. Multimedia networks

in IP networks, the same addressing scheme (IPv4/IPV6) and the same set of routing protocols (OSPF/BGP) are used for both networking modes. This implies that no address resolutions are needed when communication in one mode is triggered by an application running the other mode, and there is no duplication of routing data related software.

In ATM networks, the connection-oriented mode is well defined with a signaling protocol and a routing protocol. We recently identified several applications in ATM networks that are best supported with a connectionless mode [5]. Hence, we defined a datagram protocol called CL-ATM (Connectionless ATM) that uses the ATM End System Address (AESA) format and works in conjunction with the PNNT routing protocol. This equips ATM networks to support both networking modes using the same addressing scheme and the same routing protocol.

In summary, the assumption we make in this paper is that all three networks shown in Fig. 1 either already support or will soon support both connection-oriented and connectionless networking modes.

\section{PROPOSED INTERNETWORKING PRIN- CIPLE}

If two networks that support both connection-oriented and connectionless networking modes are to be internetworked, our proposed solution is to build a gateway that interworks the corresponding:

- User-plane protocols

- Connection-oriented mode

- Connectionless mode (datagram protocols)

- Signaling protocols

- Routing protocols

- Addressing schemes
The application of this principle to PSTN-ATM internetworking and IP-ATM internetworking is described in Section 5.

\section{EXISTING INTERNETWORKING SOLU- TIONS}

The ATM Forum standard for internetworking ATM networks with PSTNs is VTOA (Voice and Telephony Over ATM) [1], and the standard for nternetworking IP networks and ATM networks is MPOA (MultiProtocol Over ATM) [2]. This section briefly describes these two solutions in light of the internetworking principle defined in Section 3.

\subsection{VTOA (VOICE AND TELEPHONY OVER ATM)}

The ATM Forum specifications define the interworking of the user-plane protocols in the connection-oriented mode, signaling protocols and addressing schemes. Various AAL protocols have been considered for the user-plane protocol (in the connection-oriented mode) conversion from STM voice to ATM. Interworking of Q.931 signaling with Q.2931 signaling used in ATM networks is also defined. Interworking of the addressing schemes is defined by the E.164-encapsulated AESA format specified in [6]. In the current VTOA specification, there is no definition of the interworking of datagram protocols, because the CL-ATM datagram protocol is as-yet unspecified, and there is no definition of interworking of routing protocols, presumably because there are many PSTN routing protocols, none of which are official standards. MPOA (Multi-Protocol Over ATM)

The MPOA specification describes an internetworking solution for IP and ATM networks. The basis of this solution is to have IP routers with ATM interfaces perform flow detection to decide whether to send packets in a flow hopby-hop through the IP network in a connectionless mode or whether to send the packets through the ATM network in a connection-oriented mode. This is illustrated in Fig. 2. If the ATM network is to be used, an IP address to ATM address resolution is needed.

This solution is based on the assumption that the IP network only offers connectionless services, an assumption, which is no longer true, given the evolution plans to IPv6. In other words, the MPOA solution is based on an assumption that has been invalidated by recent advances in IP routers. The implication of IP routers also supporting connectionoriented networking is that if an IP router performs flow detection, it will choose to send packets from both types of flows through the IP network. Hence some other mechanism is needed to allow the router to choose between sending the data through the Internet or through the ATM network. In our networking principle, this mechanism is a result of interworking routing protocols. Without routing protocol interworking, the ATM interface on the router in the MPOA solution is a UNI (User Network Interface). In contrast, in our proposed solution, this interface is an NNI (Networkto-Network Interface), where the most significant difference between a UNI and an NNI is that a routing protocol does not 


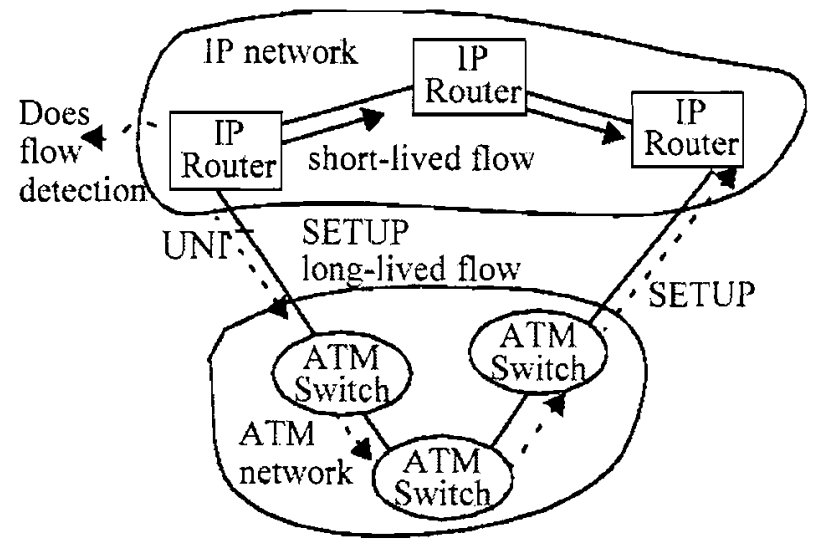

Figure 2. MPOA solution.

exist across a UNI, while it does across an NNI. Details of our proposed solution for IP-ATM internetworking is described in the next section.

\section{APPLICATION OF OUR INTERNET- WORKING PRINCIPLE}

In this section, we describe our solutions to internetwork ATM networks with the PSTN and IP networks using the principle proposed in Section 3.

\subsection{PROPOSED PSTN-ATM INTERNETWORKING SOLUTION}

By applying our general principle of internetworking to the PSTN-ATM internetworking problem, we propose building gateways that convert between the user-plane protocols, signaling protocols, routing protocols and addressing schemes used in the PSTN and those used in ATM networks. The protocols used in these networks are listed in Table 1. This gateway would convert STM voice to ATM cells, SS7 MTP L3 datagrams to CL-ATM datagrams, Q.931/ISUP signaling to UNI/PNNI [7] signaling, RTNR-like protocols to PNNI routing protocol, and E.164 addresses to AESAs. This solution is an extension of the VTOA specification to include the interworking of routing protocols and datagram protocols.

\subsection{PROPOSED IP-ATM INTERNETWORKING SOLUTION}

Fig. 3 shows our proposed solution for IP-ATM internetworking. The gateway converts between the protocols used in the IP network and those used in the ATM network as per the internetworking principle proposed in Section 3 . The protocols used in the IP network and in the ATM network are listed in Table 1.

Our solution differs from the MPOA solution described in Section 4.2 in two ways. First, internetworking in our solution is based on the use of routing information in addition to flow detection as opposed to the use of only flow detection in the MPOA solution. The reason for this difference is that the MPOA solution is based on an assumption that is no longer valid, i.e., that IP networks only support the connectionless mode. With IPv6, IP routers will be capable of differentiating flows, which in turn implies that even longlived flows can be sent hop-by-hop through IP routers. In our internetworking solution, an IP switch router sends IP packets to a gateway if the routing information indicates that this is the shortest path. For example, in Fig. 3, IP switch router I decides to send IP packets to Gateway I rather than to IP switch router II if the OSPF routing data indicates that the path through Gateway I is the more lightly-loaded path. The gateway has to accurately reflect the ATM network loading conditions and reachability data propagated by the PNNI routing protocol in its OSPF link state advertisements to IP switch router I.

Second, our solution for interworking addressing schemes is to use IP address encapsulated AESAs similar to the E.164 address encapsulated AESA format [8]. Recent standardization efforts in ISO and the ATM Forum have created such a format. This is different from the MPOA approach, which proposes using NHRP (Next Hop Resolution Protocol) [9] for address resolutions. The use of IP address encapsulated ATM address (i.e., the AESA of a node is determined automatically from its IP address) removes the need for explicit address resolutions. In MPOA, an NHRP address resolution needs to be executed prior to using the ATM connection-oriented or connectionless modes for carrying IP traffic from IP switch routers. One of the most efficient means for implementing NHRP is to place NHRP servers in IP routers and have the NHRP request proceed hopby-hop using the routing data corresponding to the IP address being resolved until the subnet in which the node whose address is being resolved is located. This implies that in all cases to use the ATM network, a small transaction needs to be executed involving the $\mathbb{P}$ network before data transmission can begin through the ATM network. If we use the transportation network analogy described in the introduction, the inefficiency of this approach becomes evident.

The gateway nodes shown in Fig. 3 encapsulate IP packets into AAL5 packets, which get segmented to ATM cells, if the connection-oriented mode of operation is desired (i.e., if a flow is used). If not, these packets are encapsulated into CL-ATM datagrams and the connectionless service in the ATM network is used. The reason for encapsulation is to simplify extracting the IP datagram at the far end (for example, Gateway II in Fig. 3) even though each layer adds an overhead from a bandwidth usage point-of-view.Based on the assumption that some applications are better served with the connectionless mode and others with the connectionoriented mode, the appropriate mode is used in the ATM network based on the type of IP packet being transported.

\section{PROTOTYPE}

We implemented our proposed IP-ATM internetworking solution as a proof of concept. There are two types of nodes in the network: (i) an ATM switch router and (ii) an IPATM gateway. The ATM switch router (ASR) consists of an 


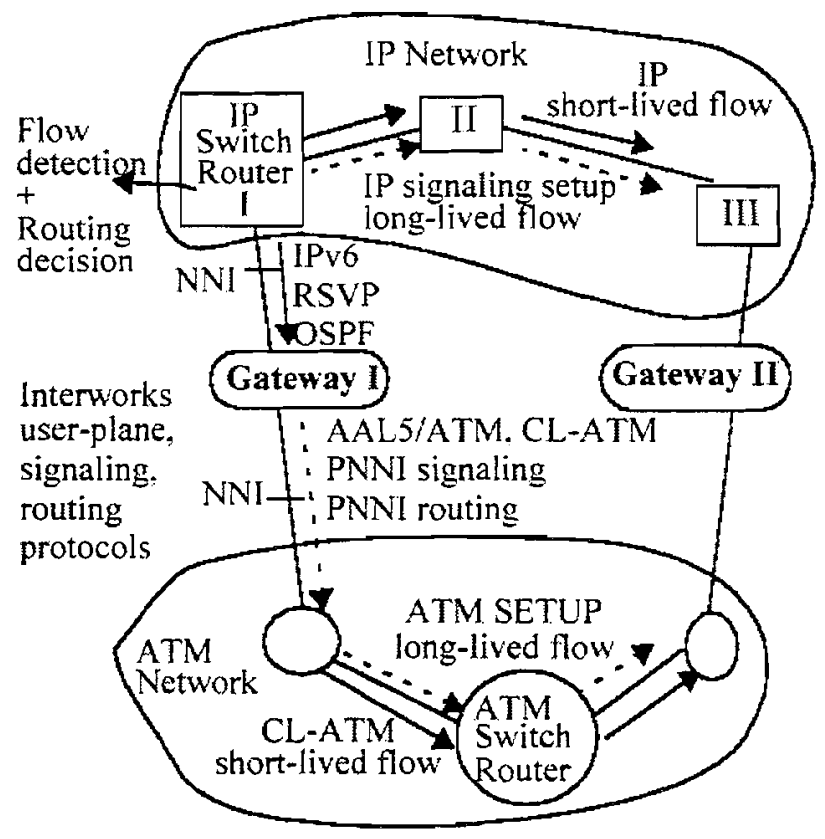

Figure 3. IP-ATM Internetworking.

ATM switch and a packet forwarding module for forwarding CL-ATM datagrams. We used two types of ATM switches, Fore Systems switches and Lucent switches. The packet forwarding module is implemented as kernel-level software on a Motorola Power PC running the PSOS operating system, which is connected to the ATM switch via a Fore Systems multimode OC-3 card. Short-lived flows are handled hop-byhop by the packet forwarding modules in the ATM switch routers, while long-lived flows are handled by the ATM switches using ATM cell forwarding.

The IP-ATM gateway hardware is a SUN workstation, running Solaris, with a Fore OC3 ATM adapter card and an ethernet interface. Two kernel-level modules are used: the IP streams module, which comes bundled with Solaris, and the CL-ATM module, which was added to the Fore ATM adapter device driver. The IP streams module is executed in router mode, i.e., it receives IP packets from the ethernet interface and sends them to the ATM interface, and vice versa. Routing data at the IP layer is set using route add system calls.

The prototype network configuration is shown in Fig. 4. The IP-ATM gateways connect the ATM network to three ethernet LANs. We use IP address encapsulated AESAs for our ATM nodes with the encapsulation method shown in Fig. 5. The subnet identifier part of the IP address is encapsulated into the peer group identifier bytes of the AESA, and the host identifier part of the IP address is encapsulated into the ESI part of the AESA. We use variable-length subnetting in our allocation of AESAs. AESAs assigned to the ATM switches are shown in Fig. 4. We obtained a subnet address 135.180.182.0 from our network administrators and created a further layer of subnets by using the first two bits of the last byte. In other words, our subnet mask is 255.255.255.192. ATM hosts attached to each switch were allocated different ESIs, but used the peer group identifier of the switch. PNNI

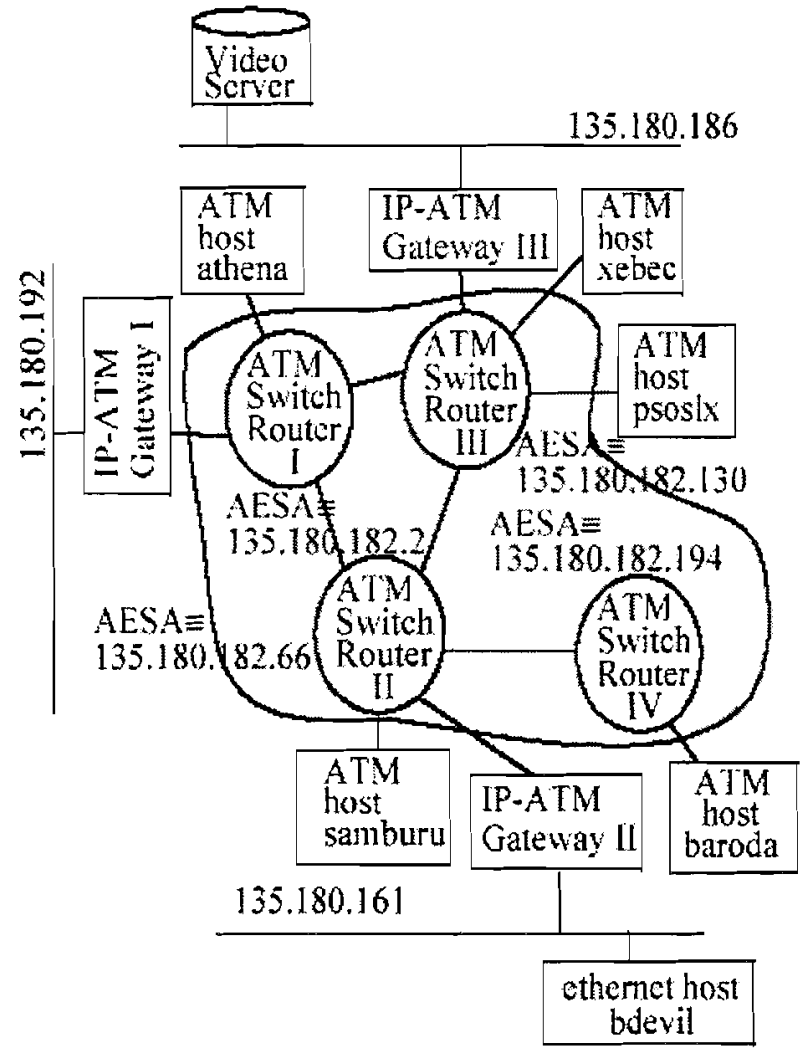

Figure 4. Network configuration.

routing tables are set up for data lookups on a mask of 10 bits (byte 6 and the first two bits of byte 7) of AESAs rather than full 20-byte address lookups. This allowed IP-ATM gateway III to announce reachability for nodes with the peer group identifier 35.0.0.35.180.186.0.0.0.0.0.0.0. IP-ATM gateways I and II similarly announce reachability for theicorresponding ethernet LAN hosts into the ATM network using PNNI topology state packets. This allows for any node on the three ethernet LANs to seamlessly communicate with each other through the ATM network and with ATM hosts. We used web browsing, telnet, the Cooltalk audio application, an MPEG video application and file transfers to demonstrate how audio, video and Internet applications can be run across IP and ATM networks.

\section{ACRONYMS}

AESA: ATM End System Address; BGP: Border Gateway Protocol; CL-ATM: ConnectionLess ATM; ISUP: ISDN User Part; NNI: Network-to-Network Interface; OSPF: Open Shortest Path First; PNNI: Private Network-to-Network Interface; PSTN: Public Switched Telephone Network; RSVP: Resource reSerVation Protocol; RTNR: Real Time Network Routing; SS7 MTP L3: Signaling System No. 7 Message Transfer Part Level 3; STM: Synchronous Time Multiplexing; STP: Signaling Transfer Point; UNI: UserNetwork Interface. 


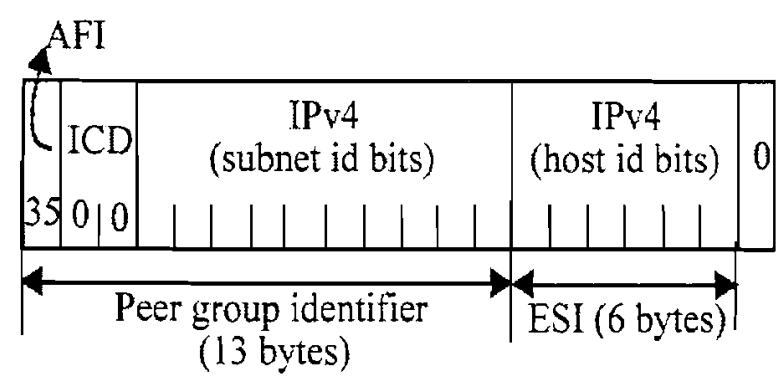

Figure 5. IP-address-encapsulated ATM End System Address (AESA) format.

\section{SUMMARY}

We presented a general internetworking principle which states that gateways are needed to interwork the userplane protocols, signaling protocols, routing protocols and addressing schemes of the two networks being internetworked. We showed how this principle can be applied to internetworking ATM networks with the PSTN and IP networks. The motivation for proposing a new solution for IP-ATM interworking is that the current ATM Forum solution for IP-ATM internetworking, MPOA, is based on an assumption that $\mathbb{P}$ networks only support the connectionless mode, an assumption that will be invalid with the introduction of IPv6, RSVP or other solutions. A feasibility study of our proposal included a prototype implementation of our IP-ATM internetworking solution.

\section{REFERENCES}

[1] ATM Forum, Voice and Telephony Over ATM to the Desktop Specification, af-vtoa-0083, Mar. '97.

[2] ATM Forum, Multi-Protocol Over ATM v1.0, BTDMPOA-MPOA-01.13, Feb. '97.

[3] S. Deering, R. Hinden, Internet Protocol, Version 6 (IPv6) Specification, RFC 1883, December 1995.

[4] R. Braden, Ed., L. Zhang, S. Berson, S. Herzog, S. Jamin, Resource ReSerVation Protocol (RSVP), RFC 2205, Sep. '97.

[5] M. Veeraraghavan, P. Pancha, and K. Y. Eng, ATM Switch Routers for Combined Connection-Oriented and Connectionless Transport, Proc. of the IEEE Globecom97, Phoenix, Arizona, Nov. 4-8, 1997.

[6] ATM Forum, ATM User-Network Interface (UNI) Signaling Specification Version 4.0, January 1996, ATM Forum/95-1434R9.

[7] ATM Fonum, Private Network-Network Spec. Interface v1.0F (PNNI 1.0), Mar. 1996.
[8] J. Bound, B. Carpenter, D. Harrington, J. Houldsworth, A. Lloyd, OSI NSAPs and IPv6, IETF RFC 1888, August 1996.

[9] D. Katz and D. Piscitello, NBMA Next Hop Resolution Protocol (NHRP), Internet Draft, May 1995.

Malathi Veeraraghavan is currently an Associate Professor in the Department of Electrical Engineering at Polytechnic University. Dr. Veeraraghavan received her BTech degree in Electrical Engineering from Indian Institute of Technology (Madras) in 1984, and MS and PhD degrees in Electrical Engineering from Duke University in 1985 and 1988, respectively. She worked for ten years in Bell Laboratories conducting research on various networking protocols and control algorithms. She holds ten patents, and has received four Best-paper awards. She served as an Associate Editor of the IEEE Transactions on Reliability from 1992 to 1994. She is currently the IEEE Communications Society E-News Editor, and an Area Editor for IEEE Communication Surveys. Currently, she also consults for Lucent Technologies. 